\title{
Quantitative circumferential strain analysis using 3-Tesla feature-tracking cardiovascular magnetic resonance in patients with old myocardial infarction
}

\author{
Ryo Ogawa ${ }^{1 *}$, Tomoyuki Kido ${ }^{1}$, Masashi Nakamura ${ }^{1,2}$, Teruhito Kido ${ }^{1}$, Akiyoshi Ogimoto ${ }^{1}$, Masao Miyagawa', \\ Teruhito Mochizuki ${ }^{1}$
}

From 19th Annual SCMR Scientific Sessions

Los Angeles, CA, USA. 27-30 January 2016

\section{Background}

Feature-tracking cardiovascular magnetic resonance (FTCMR) provides quantification of myocardial strain by analyzing cine MR images. A previous study has reported that CS measured by FT-CMR showed reasonable agreement with tagged MR in healthy volunteers. However, the usefulness of FT-CMR in patients with old myocardial infarction (OMI) has not been investigated. The purpose of this study was to evaluate diagnostic ability of CS by FT-CMR in patients with OMI.

\section{Methods}

Between March 2011 and August 2012, a total of 20 consecutive patients with OMI were enrolled in this study. All cases were performed CMR examination using a 3-Tesla MR scanner (Philips Achieva). CS by FT-CMR was analyzed using Ziostation2 (Ziosoft Inc., Tokyo, Japan). The peak subendocardial CS was quantified for 16 segments of 3 short-axis slices (basal, mid, and apical). With interobserver consensus, myocardial segments were categorized as remote normal segments $(n=173)$, adjacent segments $(n=70)$, and infarcted segments $(n=77)$ from the results of late gadolinium enhancement (LGE) with CMR. An infarcted segment was defined as an area with the presence of LGE. An adjacent segment was defined as an area adjacent to infarcted segment.

\section{Results}

The peak subendocardial CS was significantly lower in infarcted segments than in remote normal segments. $(-6.3 \pm 3.9$ vs $-11.8 \pm 3.3 ; \mathrm{p}<0.001)$. Moreover, the peak subendocardial CS was significantly lower in adjacent segments than in remote normal segments. $(-9.5 \pm$ 3.7 vs $-11.8 \pm 3.3 ; \mathrm{p}<0.05)$. A cutoff value of $-7.9 \%$ for peak subendocardial CS allowed differentiation between normal and infarcted segments with a sensitivity of $68 \%$, specificity of $75 \%$, accuracy of $73 \%$, positive predictive value of $55 \%$, negative predictive value of $84 \%$, and an area under the curve (AUC) of 0.75 .

\section{Conclusions}

FT-CMR can quantify myocardial strain without increasing examination time. Moreover, FT-CMR is useful for detecting infarcted segments.

\section{Authors' details}

'Ehime University Graduate School of Medicine, Toon, Japan. ${ }^{2}$ Saiseikai Matsuyama Hospital, Matsuyama, Japan.

Published: 27 January 2016

doi:10.1186/1532-429X-18-S1-P84

Cite this article as: Ogawa et al:: Quantitative circumferential strain analysis using 3-Tesla feature-tracking cardiovascular magnetic resonance in patients with old myocardial infarction. Journal of Cardiovascular Magnetic Resonance 2016 18(Suppl 1):P84.

${ }^{1}$ Ehime University Graduate School of Medicine, Toon, Japan

Full list of author information is available at the end of the article 


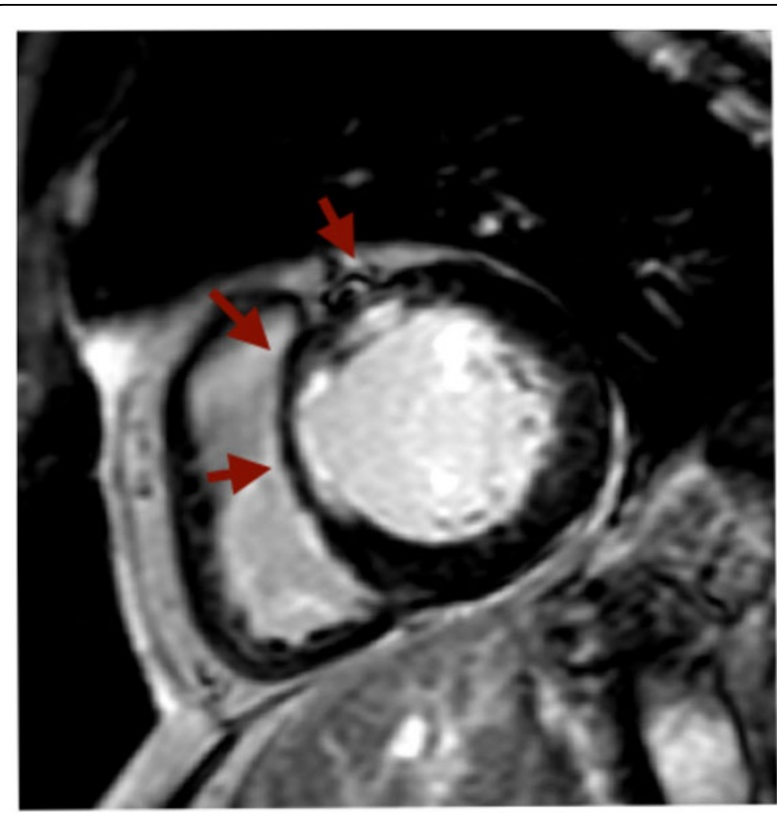

\section{Late gadolinium enhancement image}

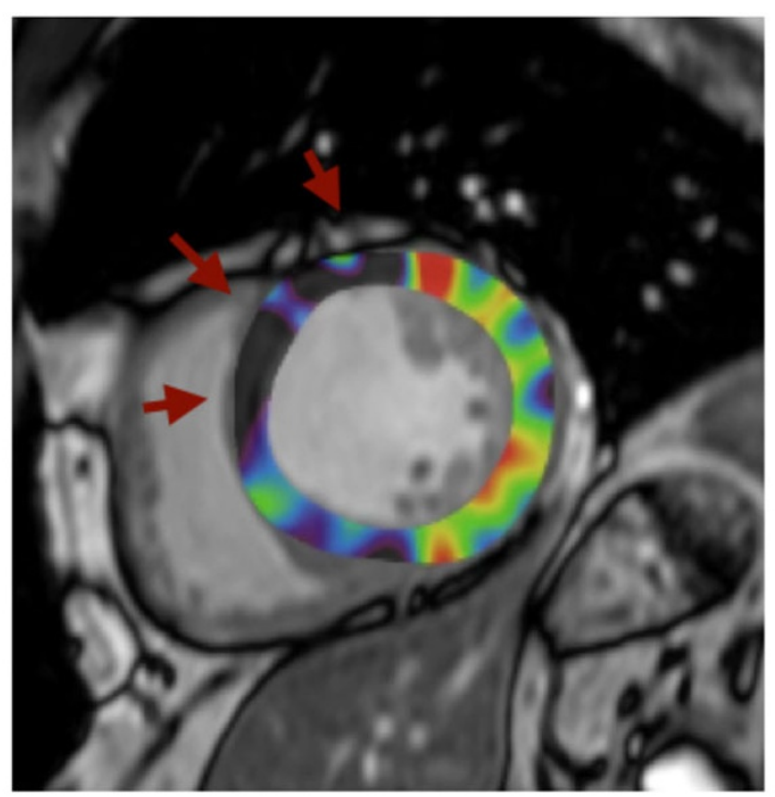

\section{Feature-tracking}

cardiovascular magnetic resonance

Figure $1 \mathrm{~A}$ short-axis late gadolinium enhancement image of a basal slice shows enhancement in the anteroseptal region. The peak subendocardial circumferential strain was decreased in anteroseptal region.

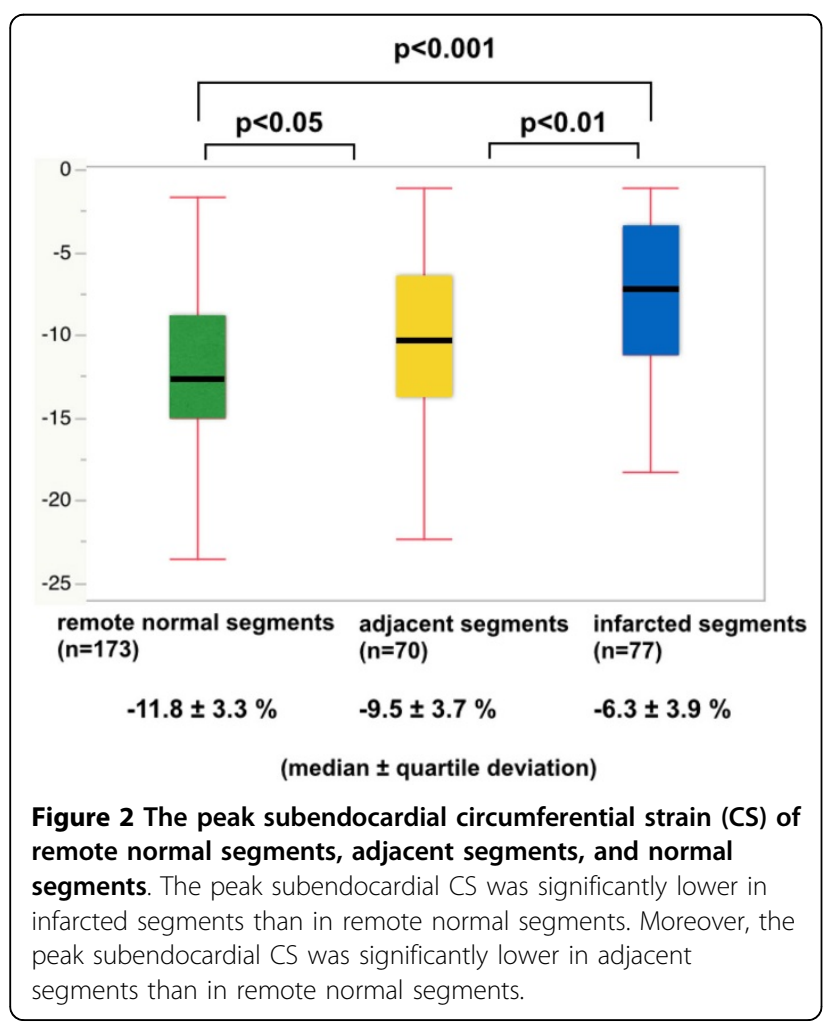

